\title{
Understanding Diversity in Health Care for a better Doctor - Patient Relationship
}

\section{Roosy Aulakh*}

Associate Professor, Department of Pediatrics, Government Medical College and Hospital, India

*Corresponding Author: Roosy Aulakh, Associate Professor, Department of Pediatrics, Government Medical College and Hospital, India.

Received: October 01, 2019; Published: November 01, 2019

DOI: $10.31080 /$ ASPE.2019.02.0175

Humans are undoubtedly diverse. No two humans are alike. They may differ in their genetic constitution, age, gender, demographic, socio-economic and ethnic background, educational and professional achievements, their lifestyle, attitudes, beliefs and perspectives and so on. Recently, there has been a growing awareness of need for diversity in health care services whereby it has been advocated that more diverse the health care providers, better customized services can be provided to the diverse patient population. A homogenous medical workforce is no longer considered apt to the diverse needs of the heterogeneous patient population. Increasing diversity in health care would help in reaching out to the underserved communities across the world. It is commonly observed that patients prefer approaching a medical professional with whom he/she can relate to in terms of gender/ethnicity/religious or political beliefs. There is an inbuilt trust for such a health professional in the minds of the patient and goes a long way in building long lasting healthy doctor-patient relationship.

Competence of a health professional in dealing with diverse patient population is not only limited to knowledge of ethnic variations in a disease but also in understanding the beliefs of the patient community thereby tailoring his medical advice in a way which is more easily acceptable to the patients. Only if the patient trusts the doctor and is convinced with the medical advice will he/ she be compliant with the medical advice and/or treatment.

An unexplored aspect of the diversity in health care is spreading awareness amongst the patients of diversity in professional approach of various health care providers. It is not uncommon to witness patients coming up with a preformed notion about how a medical professional should approach the patient's health problem. Some patients feel that clinical observation should suffice and costly investigations should not be resorted to while at the other extreme end are patients who feel as if the doctor didn't offer a comprehensive medical checkup in absence of detailed investigations. For the same problem, some patients would insist for outpatient care while others would feel anxious unless inpatient services are offered. This understanding and acceptance of diversity among health care providers in their professional approach would only be possible if the patients trust their health care providers and understand that each health care provider works to improve the medical condition of the patient but may have a diverse approach than considered 'professional attitude' by the patient. Such healthy relationship built on foundation of strong trust and acceptance of diversity in not only the patient population but also the health care professionals' approach to such diverse patients would go a long way in improving the ever-deteriorating doctor-patient relationship.

\section{Volume 2 Issue 12 December 2019 (C) All rights are reserved by Roosy Aulakh.}

